\title{
Absence of Wolbachia endobacteria in the non-filariid nematodes Angiostrongylus cantonensis and A. costaricensis Jeremy M Foster ${ }^{1}$, Sanjay Kumar ${ }^{1}$, Louise Ford ${ }^{2}$, Kelly L Johnston ${ }^{2}$, Renata Ben ${ }^{3}$, Carlos Graeff-Teixeira ${ }^{3}$ and Mark J Taylor*2
}

\author{
Address: ${ }^{1}$ Parasitology Division, New England Biolabs, 240 County Road, Ipswich, MA 01938, USA, ${ }^{2}$ Molecular and Biochemical Parasitology \\ Liverpool School of Tropical Medicine, Pembroke Place, Liverpool L3 5QA, UK and ${ }^{3}$ Laboratório de Parasitologia Molecular, Instituto de Pesquisas \\ Biomédicas da PUCRS, Porto Alegre, Brazil \\ Email: Jeremy M Foster - foster@neb.com; Sanjay Kumar - kumar@neb.com; Louise Ford - lford@liverpool.ac.uk; \\ Kelly L Johnston - johnkel@liverpool.ac.uk; Renata Ben - benrenata@gmail.com; Carlos Graeff-Teixeira - graeff.teixeira@gmail.com; \\ Mark J Taylor* - mark.taylor@liverpool.ac.uk \\ * Corresponding author
}

Published: 18 September 2008

Parasites \& Vectors 2008, I:31 doi:|0.|| |86/|756-3305-|-3|
Received: 17 July 2008

Accepted: 18 September 2008

This article is available from: http://www.parasitesandvectors.com/content/I/I/3I

(C) 2008 Foster et al; licensee BioMed Central Ltd.

This is an Open Access article distributed under the terms of the Creative Commons Attribution License (http://creativecommons.org/licenses/by/2.0), which permits unrestricted use, distribution, and reproduction in any medium, provided the original work is properly cited.

\begin{abstract}
The majority of filarial nematodes harbour Wolbachia endobacteria, including the major pathogenic species in humans, Onchocerca volvulus, Brugia malayi and Wuchereria bancrofti. These obligate endosymbionts have never been demonstrated unequivocally in any non-filariid nematode. However, a recent report described the detection by PCR of Wolbachia in the metastrongylid nematode, Angiostrongylus cantonensis (rat lungworm), a leading cause of eosinophilic meningitis in humans. To address the intriguing possibility of Wolbachia infection in nematode species distinct from the Family Onchocercidae, we used both PCR and immunohistochemistry to screen samples of $A$. cantonensis and $A$. costaricensis for the presence of this endosymbiont. We were unable to detect Wolbachia in either species using these methodologies. In addition, bioinformatic and phylogenetic analyses of the Wolbachia gene sequences reported previously from $A$. cantonensis indicate that they most likely result from contamination with DNA from arthropods and filarial nematodes. This study demonstrates the need for caution in relying solely on PCR for identification of new endosymbiont strains from invertebrate DNA samples.
\end{abstract}

\section{Findings}

Wolbachia endobacteria infect most species of insect and are present in other arthropod groups as well as in most filarial nematode species. Phylogenetic analyses currently indicate as many as eight distinct Wolbachia lineages, designated supergroups A to $\mathrm{H}$, along with some other lineages whose taxonomic position remains unresolved $[1,2]$. Arthropod Wolbachia are found in all supergroups except $\mathrm{C}$ and $\mathrm{D}$, with the majority of insect Wolbachia strains in supergroups A and B. Wolbachia from filarial nematodes are exclusively in supergroups $\mathrm{C}$ and $\mathrm{D}$ with the exception of endosymbionts from Mansonella spp., which are in supergroup F along with the Wolbachia from certain termites.

The association between Wolbachia and filarial nematodes appears to be one of mutualism, probably of an obligatory nature [3]. Elimination of the endosymbionts by antibiotic treatment disrupts embryogenesis and hence microfilarial production, disrupts growth and development, and leads to macrofilaricidal effects. Wolbachia are also implicated in the immunopathology of infected per- 
sons and may contribute to the inflammatory adverse events seen after standard anti-filarial chemotherapy [3]. Thus, targeting the Wolbachia endosymbiont has emerged as an attractive new strategy for filarial disease control [3].

Until recently, despite extensive investigation of diverse nematode groups, there had been no identification of Wolbachia in any non-filariid nematode [4,5]. However, Wolbachia ftsZ, wsp (Wolbachia surface protein) and 16S rDNA sequences were recently amplified by PCR from DNA preparations of the metastrongylid nematode, Angiostrongylus cantonensis [6]. Based on phylogenetic analysis of the $w s p$ sequence, the apparent endosymbiont from $A$. cantonensis appeared to have a lineage distinct from the filarial Wolbachia (supergroups C, D or F) and was tentatively positioned in supergroup G, containing the Wolbachia from certain spiders such as Diaea circumlita. Both A. cantonensis and A. costaricensis are occasional pathogens of humans, the former a leading cause of eosinophilic meningitis in Asia and Pacific Islands while the latter produces abdominal disease in the Americas $[7,8]$. The unexpected detection of Wolbachia in A. cantonensis and the medical implications of targeting this endosymbiont for novel antibiotic therapies for control of eosinophilic meningitis prompted us to investigate the status of Wolbachia in the genus Angiostrongylus in more detail.

A. cantonensis was obtained from Prof. Kentaro Yoshimura and maintained as a laboratory life-cycle in the Department of Parasitology of the Akita University Medical School. A. costaricensis strain "Santa Rosa" has been maintained in the Laboratório de Parasitologia Molecular, Instituto de Pesquisas Biomédicas da PUCRS since 1992 (in mice and the wild rodent Oligoryzomis nigripes; veronicelid slugs and Biomphalaria glabrata snails). Adult Brugia malayi (TRS labs) were used as a positive control for PCR and immunohistochemistry

PCR analysis of Wolbachia genes from both A. cantonensis and $A$. costaricensis adult worms showed no evidence that either species harbours Wolbachia endosymbionts. Genomic DNA from individual adult female A. cantonensis and A. costaricensis, which had been stored in $80 \%$ ethanol, was isolated (QiaAmp ${ }^{\circledR}$ DNA mini kit, Qiagen) and analysed for Wolbachia by PCR as previously described [6] with modifications. PCR was carried out on a iCycler thermocycler (Bio-Rad) using the following conditions: $95^{\circ} \mathrm{C}$ for $4 \mathrm{~min}$, followed by 40 cycles of $94^{\circ} \mathrm{C}$ for $15 \mathrm{~s}, 48^{\circ} \mathrm{C}$ for $30 \mathrm{~s}, 72^{\circ} \mathrm{C}$ for $2 \mathrm{~min}$, then $72^{\circ} \mathrm{C}$ for $10 \mathrm{~min}$, using primer pairs widely used for detection of Wolbachia from diverse hosts, and previously used on DNA from A. cantonensis [6]. The following primers were used for amplification of Wolbachia gene sequences: wsp (wsp81F; 5'-TGG TCC AAT AAG TGA TGA AGA AAC-3' and wsp691R; 5'AAA AAT TAA ACG CTA CTC CA-3'); ftsZ (ftsZ357F; 5'-
CAA AAA TAT GTG GAT ACG CTC ATT GT-3' and ftsZ788R; 5'-GTA GCA CCA AAT ATT ATA TTT GCA TTT TC-3'); and 16S rRNA (16SwolbF; 5'-GAA GAT AAT GAC GGT ACT CAC-3' and 16SwolbR3; 5'-GTC ACT GAT CCC ACT TTA AAT AAC-3'). PCR reactions were performed in $25 \mu \mathrm{l}$ containing $1 \mu \mathrm{l}$ gDNA, $0.3 \mu \mathrm{M}$ of each primer, 0.2 $\mathrm{mM}$ dNTPs, $1.5-3.0 \mathrm{mM} \mathrm{MgCl}_{2}$ and $0.625 \mathrm{U}$ of Taq polymerase (New England Biolabs [NEB]) in $1 \times$ reaction buffer (NEB). Positive PCR amplification was shown using DNA isolated from individual adult female $B$. malayi, which are known to contain Wolbachia, thus demonstrating that the primers and conditions were optimal for Wolbachia detection. All DNA samples produced nematode specific gene products. Angiostrongylus 18S rRNA, based on GenBank sequences from $A$. cantonensis and $A$. costaricensis ([GenBank:AY295804] and [GenBank:EF514913], respectively) was amplified with primers Ac18S 30F; 5'-AAG TGA AAC TGC GAA CGG CT-3' and Ac18S 830R; 5'-TCA CCT CTC GCG CAG GGA TA-3', while $B$. malayi gst was amplified as previously described [9] using GST 1377; 5'-TGC TCG CAA ACA TAG TAA TAG T-3' and GST 1632; 5'-ATC ACG GAC GCC TTC ACA G-3', indicating that there was DNA at sufficient concentrations for detection by standard one-round PCR.

In order to detect Wolbachia by immunohistochemistry, $A$. cantonensis and A. costaricensis worms were fixed in $80 \%$ ethanol and embedded in paraffin blocks. Sections were stained using affinity purified anti-Wolbachia peptidoglycan-associated lipoprotein (WoLP) antibodies and rabbit polyclonal anti-sera raised to Wolbachia surface protein (WSP) and visualized using the UltraVision ONE detection system (Lab Vision, ThermoFisher Scientific) using haematoxylin as a counterstain. In contrast to the positive staining observed in sections of B. malayi, both Angiostrongylus species were found to be negative with each of the Wolbachia-specific antibodies or antisera. It should be noted that both of these reagents are able to detect Wolbachia in species as diverse as filarial nematodes and the mosquito Aedes albopictus [[9-11], M. Taylor, unpublished data], indicating that a lack of cross-reactivity to Wolbachia found in Angiostrongylus is unlikely.

Because we were unable to reproduce the detection of Wolbachia in Angiostrongylus spp by PCR or immunohistochemistry, we analyzed in detail the sequences deposited in the GenBank database as part of the earlier report [6]. Comparison of the wsp nucleotide sequence [GenBank:AY508980] to the non-redundant nucleotide database (nr) using BLASTN revealed good identity (97\%) to the wsp sequence [GenBank:AY486092] from the putative supergroup G Wolbachia from the spider, D. circumlita c2, as described previously [6]. However, the best identity (99\%) was to wsp from the Wolbachia of the mosquito, Malaya genurostris [GenBank:AY462865]. Wolbachia mul- 
tilocus sequence typing (MLST) has shown that phylogenetic inference based upon wsp sequences yields spurious lineages due to the high levels of intragenic recombination in this gene [1]. MLST of the Wolbachia of the spider, D. circumlita $\mathrm{c} 2$, indicates that this endosymbiont is more correctly a member of supergroup A [1], and so too presumably is the Wolbachia from the mosquito, M. genurostris.

We performed similar nucleotide comparisons of the 16S [GenBank:AY652762] and ftsZ [GenBank:DQ159068] sequences attributed to Wolbachia from A. cantonensis and constructed phylogenetic trees for each. Wolbachia sequences used for multiple alignments and phylogenetic tree construction are as follows, with the first GenBank sequence for each invertebrate host organism corresponding to $16 \mathrm{~S}$ and the second to ftsZ: Brugia malayi [GenBank:AJ010275], [GenBank:AJ010269]; $\underline{B}$. pahangi [GenBank:AJ012646], [GenBank:AJ010270]; Litomosoides sigmodontis [GenBank:AF069068], [GenBank:AJ010271]; Onchocerca volvulus [GenBank:CU062464], GenBank:AJ276501]; O. gutturosa [GenBank:AJ276498], [GenBank:AJ010266]; Dirofilaria immitis [Genbank:Z49261], [GenBank:AJ010272]; Drosophila melanogaster [GenBank: NC 002978.6; genome coordinates: 1167943-1169389], [GenBank:U28189]; ㅁ. simulans wRiverside [GenBank:DQ412085], [GenBank:U28178]; Trichogramma cordubensis [GenBank:L02883], [GenBank:U28200]; Culex pipiens [Genbank:U23709], [GenBank:U28209]; Folsomia candida [GenBank:AF179630], [GenBank:AJ344216]; Kalotermes flavicollis [GenBank:Y11377], [GenBank:ㅍ292345], Mansonella perstans 16S [GenBank:AY278355]; Mansonella sp. ftsZ [GenBank:AJ628414]. The underlined characters for each host species represent the abbreviations shown in the trees. The 16S sequence attributed to Wolbachia from A. cantonensis had highest nucleotide identity (99\%) to Wolbachia $16 \mathrm{~S}$ from D. immitis [GenBank:Z49261]. This identity was better than that between Wolbachia 16S sequences from sister species within the genus Dirofilaria, namely D. immitis and D. repens $(98 \%)$. An equal match was detected for Wolbachia apparently from an engorged dog tick, Rhipicephalus sanguineus [GenBank:AF304445], but since this tick lacks Wolbachia [12], we propose that this Wolbachia sequence derived from $D$. immitis acquired during a blood feed on a heartworm-infected dog. The $16 \mathrm{~S}$ phylogenetic tree resolved supergroups A to $\mathrm{F}$ and confirmed the near identity of the sequence amplified from A. cantonensis to the Wolbachia $16 \mathrm{~S}$ from D. immitis (Figure 1a; see additional file 1: Wolbachia 16S multiple sequence alignment - A. cantonensis). The available 16S sequences for Wolbachia from $A$. cantonensis and from $D$. circumlita are partial gene sequences and have very little overlap with each other. Therefore, we were unable to include D. circumlita Wolbachia 16S in the same alignment and phylogenetic tree as the 16S reported from Wolbachia from A. cantonensis. However, a separate alignment and tree using 16S fragments corresponding to that from Wolbachia of D. circumlita showed that this sequence is quite distinct from the $D$. immitis Wolbachia $16 \mathrm{~S}$ and clusters with sequences from Wolbachia of arthropods (Figure 1b; see additional file 2: Wolbachia 165 multiple sequence alignment - D. circumlita), as expected based on recent Wolbachia MLST [1]. Therefore, while the $w s p$ gene reported for Wolbachia from A. cantonensis has high identity to sequences from the endosymbionts of the arthropods, M. genurostris and $D$. circumlita, the $16 \mathrm{~S}$ sequence is nearly identical to Wolbachia 16S from filarial nematodes, notably D. immitis (supergroup C). The results of our analyses of fts $Z$ were very similar to those obtained for $16 \mathrm{~S}$. We observed $99 \%$ identity to the ftsZ reported from the Wolbachia from $D$. immitis [GenBank:AJ010272]. The level of identity between these two sequences also exceeded that between the Wolbachia fts $Z$ sequences from the sister species $D$. immitis and D. repens (92\%). A phylogenetic analysis of $f t s Z$ sequences representing Wolbachia supergroups A to F (Figure 1c; see additional file 3: Wolbachia ftsZ multiple sequence alignment) also resolved these six groups and confirmed the high similarity of the sequence reported for Wolbachia from A. cantonensis and that from D. immitis.

In conclusion, our inability to detect Wolbachia in two different species of Angiostrongylus by PCR or immunohistochemistry argues against the presence of this endosymbiont in these metastrongylid nematodes. Lateral gene transfers from Wolbachia to invertebrates are common [13]. Such a phenomenon could conceivably have resulted in the presence of Wolbachia fragments in the A. cantonensis genome, but since we were unable to detect Wolbachia sequences using the same PCR primers as were used in the earlier report [6], this possibility seems most unlikely. Instead, we suspect contamination of the DNA samples or PCR reactions with Wolbachia DNA from the mosquito, M. genurostris in the case of wsp and the filarial nematode, D. immitis, in the case of both ftsZ and 16S. This seems very plausible since the nucleotide identity is $99 \%$ in all cases. In support of this conclusion, we note that the wsp sequence of the $\mathrm{M}$. genurostris endosymbiont was deposited in the GenBank database by the same authors of the recent report on Wolbachia in A. cantonensis, and that they carry out research on D. immitis. We cannot rule out the possibility that $\mathrm{A}$. cantonensis from Taiwan contain Wolbachia while the worms we analyzed from Japan do not. However, the high identities of the reported sequences to those from arthropod Wolbachia (supergroup A) on the one hand, but to the Wolbachia from the nematode, D. immitis (supergroup C), on the other, evoke either a double Wolbachia infection, a phenomenon never observed in nematode-Wolbachia symbioses, or a highly divergent Wolbachia lineage unlike any 

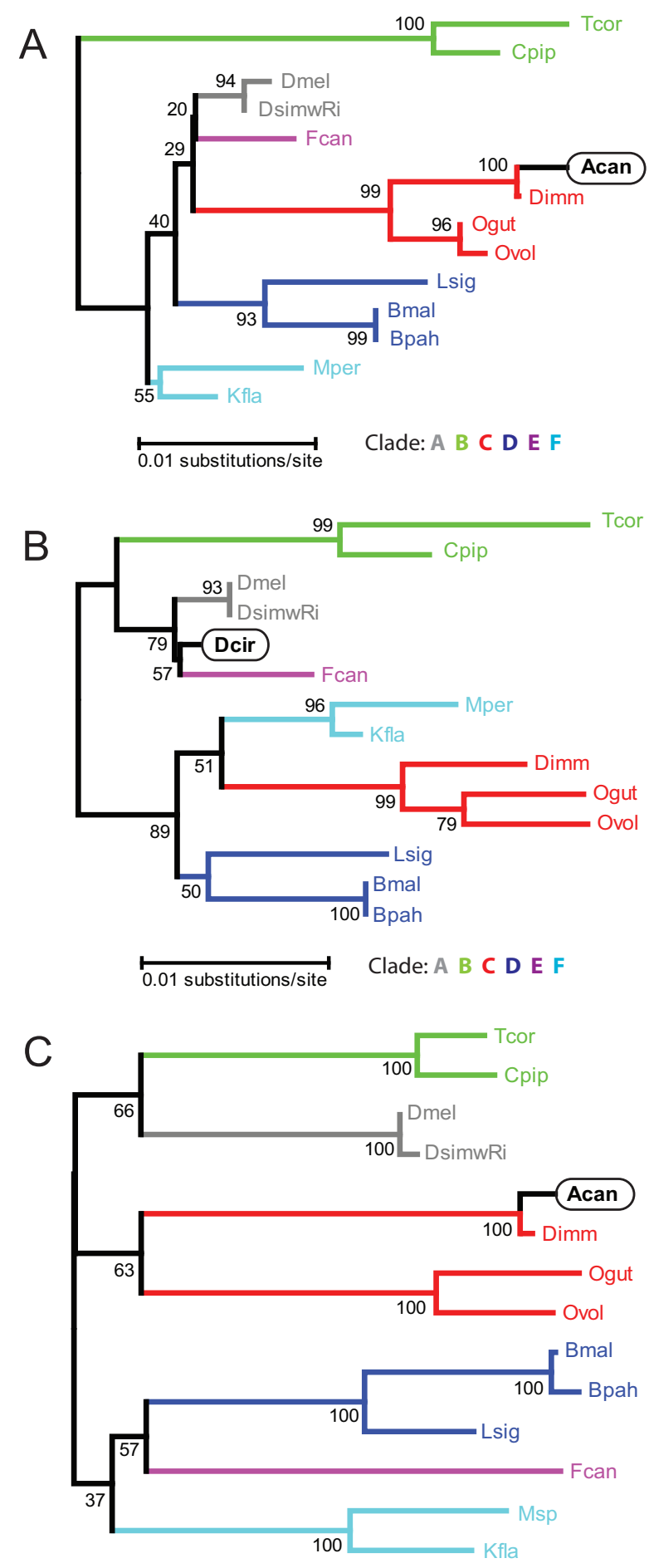

0.01 substitutions/site Clade: A B C D E F

\section{Figure I}

Minimum evolution trees based on alignments of $A$ ) the Wolbachia I 6S (770 nucleotides) reported from $A$. cantonensis [GenBank:AY652762], B) the Wolbachia I6S (639 nucleotides) of $D$. circumlita c2 [GenBank:AY486072], and C) the Wolbachia ftsZ (43 I nucleotides) reported from $\underline{A}$. cantonensis [GenBank:DQI59068 ]. Sequences were aligned using ClustalX version 2.0.7 [14] using default parameters for slow/accurate alignment [Gap Opening: 10, Gap Extend: 0. I, IUB DNA weight matrix]. After alignment, sequences were manually trimmed to the endpoints of the $16 \mathrm{~S}$ sequences of the Wolbachia from $A$. cantonensis (see additional file I: Wolbachia I6S multiple sequence alignment $-A$. cantonensis) and $D$. circumlita (see additional file 2: Wolbachia I6S multiple sequence alignment $-D$. circumlita), and to the endpoints of the fts $Z$ sequence of the Wolbachia from $A$. cantonensis (see additional file 3: Wolbachia ftsZ multiple sequence alignment). Phylogenetic trees were calculated using the Minimum Evolution method in MEGA4 [15]. The percentage of replicate trees in which the associated taxa clustered together in the bootstrap test (I000 replicates) is shown next to the branches. Evolutionary distances were computed using the Maximum Composite Likelihood. The Minimum Evolution tree was searched using the Close-Neighbor-Interchange (CNI) algorithm at a search level of I. The Neighbor-joining algorithm was used to generate the initial tree. All positions containing gaps and missing data were eliminated from the dataset (Complete deletion option).

reported thus far. The most straightforward conclusion from our analysis is that Angiostrongylus sp. do not contain Wolbachia.

\section{Competing interests}

The authors declare that they have no competing interests.

\section{Authors' contributions}

MT and JF wrote the paper. JF and SK performed bioinformatic analyses and constructed the multiple sequence alignments and phylogenetic trees. LF carried out PCR analyses and $\mathrm{KJ}$ immunohistochemistry and both contributed to writing the paper. RB and C G-T performed the initial PCR analysis and provided parasite material. MT conceived and directed the study. All authors read and approved the final manuscript.

\section{Figure I}




\section{Additional material}

\section{Additional file 1}

Wolbachia 165 multiple sequence alignment - A. cantonensis. A multiple sequence alignment of the $16 S$ sequence attributed to Wolbachia from A. cantonensis and corresponding $16 S$ fragments from Wolbachia from diverse arthropod and nematode hosts.

Click here for file

[http://www.biomedcentral.com/content/supplementary/17563305-1-31-S1.pdf]

\section{Additional file 2}

Wolbachia 165 multiple sequence alignment - D. circumlita. A multiple sequence alignment of the $16 S$ sequence of Wolbachia from $\mathrm{D}$. circumlita and corresponding $16 S$ fragments from Wolbachia from diverse arthropod and nematode hosts.

Click here for file

[http://www.biomedcentral.com/content/supplementary/17563305-1-31-S2.pdf]

\section{Additional file 3}

Wolbachia ftsZ multiple sequence alignment. A multiple sequence alignment of the ftsZ sequence attributed to Wolbachia from A. cantonensis and corresponding ftsZ fragments from Wolbachia from diverse arthropod and nematode hosts.

Click here for file

[http://www.biomedcentral.com/content/supplementary/17563305-1-31-S3.pdf]

\section{Acknowledgements}

We thank the Bill and Melinda Gates Foundation for support of the A-WOL consortium (MT, LF, KJ \& SK) and New England Biolabs for financial support (JF, SK).

\section{References}

I. Baldo L, Werren JH: Revisiting Wolbachia supergroup typing based on WSP: spurious lineages and discordance with MLST. Curr Microbiol 2007, 55:81-87.

2. Casiraghi M, Bordenstein SR, Baldo L, Lo N, Beninati T, Wernegreen J, Werren JH, Bandi C: Phylogeny of Wolbachia pipientis based on gltA, groEL and ftsZ gene sequences: clustering of arthropod and nematode symbionts in the $F$ supergroup, and evidence for further diversity in the Wolbachia tree. Microbiology 2005, I 5 I:40I5-4022.

3. Taylor MJ, Bandi C, Hoerauf A: Wolbachia bacterial endosymbionts of filarial nematodes. Adv Parasitol 2005, 60:245-284.

4. Bordenstein SR, Fitch DHA, Werren JH: Absence of Wolbachia in nonfilariid nematodes. J Nematol 2003, 35:266-270.

5. Duron O, Gavotte L: Absence of Wolbachia in Nonfilariid Worms Parasitizing Arthropods. Curr Microbiol 2007, 55:193-197.

6. Tsai KH, Huang CG, Wang LC, Yu YW, Wu WJ, Chen WJ: Molecular evidence for the endosymbiont Wolbachia in a non-filaroid nematode, Angiostrongylus cantonensis. J Biomed Sci 2007, | 4:607-615.

7. Graeff-Teixeira C, Camillo-Coura L, Lenzi HL: Clinical and epidemiological aspects of abdominal angiostrongyliasis in southern Brazil. Rev Inst Med Trop Sao Paulo I991, 33:373-378.

8. Pien FD, Pien BC: Angiostrongylus cantonensis eosinophilic meningitis. Int J Infect Dis 1999, 3:161-163.

9. McGarry HF, Egerton GL, Taylor MJ: Population dynamics of Wolbachia bacterial endosymbionts in Brugia malayi. Mol Biochem Parasitol 2004, 135:57-67.
10. McGarry HF, Pfarr K, Egerton G, Hoerauf A, Akue JP, Enyong P, Wanji S, Klager SL, Bianco AE, Beeching NJ, Taylor MJ: Evidence against Wolbachia symbiosis in Loa loa. Filaria J 2003, 2:9.

II. Turner JD, Langley RS, Johnston KL, Egerton G, Wanji S, Taylor MJ: Wolbachia endosymbiotic bacteria of Brugia malayi mediate macrophage tolerance to TLR- and CD40-specific stimuli in a MyD88/TLR2-dependent manner. J Immunol 2006, 177:1240-1249.

12. Noda H, Munderloh UG, Kurtti TJ: Endosymbionts of ticks and their relationship to Wolbachia spp. and tick-borne pathogens of humans and animals. Appl Environ Microbiol 1997, 63:3926-3932.

13. Hotopp JC, Clark ME, Oliveira DC, Foster JM, Fischer P, Torres MC, Giebel JD, Kumar N, Ishmael N, Wang S, Ingram J, Nene RV, Shepard J, Tomkins J, Richards S, Spiro DJ, Ghedin E, Slatko BE, Tettelin H, Werren $\mathrm{JH}$ : Widespread lateral gene transfer from intracellular bacteria to multicellular eukaryotes. Science 2007, 3 I 7:1753-1756.

14. Larkin MA, Blackshields G, Brown NP, Chenna R, McGettigan PA, McWilliam H, Valentin F, Wallace IM, Wilm A, Lopez R, Thompson JD, Gibson TJ, Higgins DG: Clustal $W$ and Clustal $X$ version 2.0. Bioinformatics 2007, 23:2947-2948.

15. Tamura K, Dudley J, Nei M, Kumar S: Molecular Evolutionary Genetics Analysis (MEGA) software version 4.0. Mol Biol Evol 2007, 24:1596-1599.
Publish with Bio Med Central and every scientist can read your work free of charge

"BioMed Central will be the most significant development for disseminating the results of biomedical research in our lifetime. "

Sir Paul Nurse, Cancer Research UK

Your research papers will be:

- available free of charge to the entire biomedical community

- peer reviewed and published immediately upon acceptance

- cited in PubMed and archived on PubMed Central

- yours - you keep the copyright
BioMedcentral 